GRADUATE THESIS REPORT

\title{
iTVCare: A home care system for the elderly through interactive television
}

\author{
Pedro C. Santana-Mancilla, Luis E. Anido-Rifón
}

Published: 31 October 2018

\begin{abstract}
In the world, many older adults are living alone for most of the day. This work propose the use of the television set, a medium well known by seniors, to improve the way that seniors and their caregivers can track daily activities such as medication intakes reminders. Two evaluations were held, a heuristic usability assessment early on the design process and an evaluation of the technology adoption. Both evaluations generated initial evidence that the system supported elders in achieving a better quality of life.
\end{abstract}

Keywords: Interactive television; seniors; elderly; homecare; technology adoption; public nursing homes.

\section{Introduction}

In Mexico, many seniors are alone for most of the day or live in public nursing homes. Aging is related to the accumulation (during the lifetime elapsed) of genetic faults in cells and organs; in some cases, augmented by additional factors, such as: diet, lifestyle and diseases [3]. It is considered, that for elders, is necessary to provide simple interaction with computer systems [7]. They often left outside technological tools because these were not designed to support the natural changes that older adults face due to ageing [6], and elderly people typically show rejection of new technologies at home, either because the lack of support to their natural disabilities or the lack of perceived utility [5]. This thesis proposes to explore a medium well known by seniors such as the television set (TV). TV is a standard device with an extensive home penetration and impacts in practically all scopes from the point of view of the information, entertainment and education $[10,11]$.

\section{Related work}

Support for disease and aging has been a widely researched topic. Next, we describe some solutions that support, through television, seniors living alone in their home.

\footnotetext{
Santana-Mancilla, PC. ${ }^{1,2}$, Anido-Rifón, LE. ${ }^{1}$

${ }^{1}$ School of Telecommunications Engineering

University of Vigo

Vigo, Spain

Email: psantana@uvigo.es, lanido@gist.uvigo.es

${ }^{2}$ School of Telematics

University of Colima

Colima, Mexico
}

\subsection{Nurse Gudrun}

This project is an IPTV-based solution that allows patients to communicate with the hospital through an interactive television channel. The patients are equipped with set-top boxes for their televisions and with a broadband connection to use the special channel by means of the remote control and a webcam.

Patients can be seen on the screen themselves and their doctor at the same time as they talk, and the camera can zoom in so that the doctor can see more details of the patient's condition [4].

\subsection{AAL FoSSIBLE}

This is a project to support social interactions through the development of smart TV applications to prevent isolation for older people living alone [2].

The use of television to provide help to elders is only emerging, as reported in [5], which queried Scopus and Web of Science for papers using keywords "Elderly \& TV", "Interactive \& TV \& Elderly", "SmartHome", "AAL \& elderly \& TV" and filtering only those related to elderly older people and television, more than $75 \%$ of publications were released at or after 2010 .

As can be seen, there are different approaches and proposals in the literature to face the problem, in addition, if we take into consideration that elders think that using technology is very complex, we must rethink the process using familiar forms of interaction to them, in order to make it comfortable, hence the importance of validating the usability and the technology acceptance.

\section{Research Objective}

The main objective of this research is design, development and evaluation of a home care platform for older adults using interactive television technologies in order to improve their technology adoption. To achieve this, we propose the next specific objectives:

- Understanding older adults needs.

- Requirements specification.

- Identify appropriate technologies for the development of interactive television applications.

- Field evaluation of the platform.

\section{Methods}

This research used the User Centered Design [1], which is detailed below. Specify the usage context: Identify people that will use the product, what their goals are in using it, and under which circumstances they will use it. Specify requirements: 


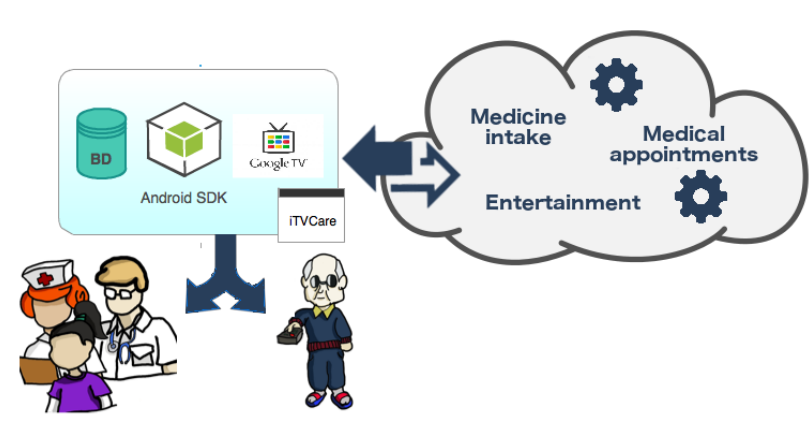

Figure 1. iTVCare cloud architecture.

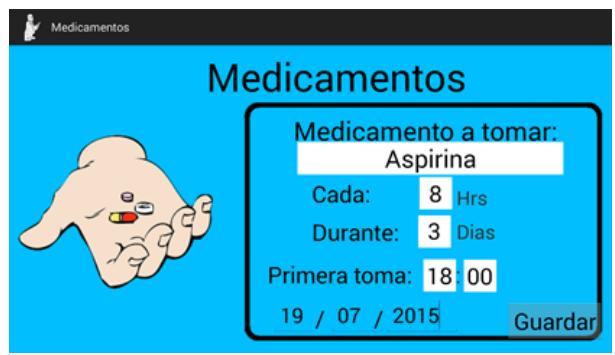

Figure 2. iTVCare, Screen to add the medicine intake reminder. Source [9].

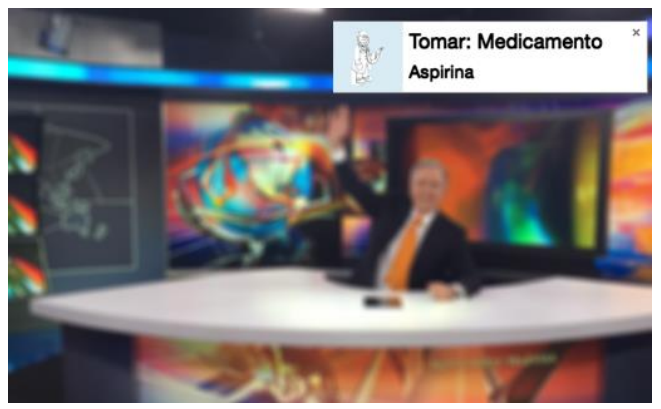

Figure 3. Notification of the reminder while the older adults are watching TV. Source [9].

Table 1. Characteristics of the expert evaluators

\begin{tabular}{|c|c|c|c|c|}
\hline Expert & Gender & Age & Education & Occupation \\
\hline 1 (pilot) & F & 30 & Masters & Graduated student \\
\hline 2 & M & 34 & Masters & Researcher \\
\hline 3 & M & 34 & Masters & Researcher \\
\hline 4 & F & 39 & Doctorate & Researcher \\
\hline 5 & M & 41 & Doctorate & Researcher \\
\hline 6 & M & 24 & Bachelor & Graduated student \\
\hline
\end{tabular}

Identify all the requirements and the users' objectives that are needed for success with the product. Produce design solutions: This part must follow an iterative process, starting from a low fidelity prototype to a high-fidelity prototype. Evaluation: This stage must be (ideally) executed through tests with real users; this is the most important part of the process.

\section{Current Results}

\subsection{Contextual study}

The context usage was gathered based on surveys with 500 older adults (65+ years) in the Mexican state of Colima. Some of the relevant results were [9]:
- The television sets have a high percentage of penetration in Mexican homes, 99\% percent of the interviewed mentioned having one.

- TV was widely used as an important part of daily activities, $85.86 \%$ watched TV for more than an hour a day, and $37.17 \%$ spent more than three hours watching television.

\subsection{Prototype design}

By analyzing the needs gathered from the surveys and considering the literature about the design of user interfaces for older adults, a low-fidelity prototype was designed. The prototype was called iTVCare and these are its main features (see Figure 1):

- Minimize the number of steps from the home screen to reach a given screen.

- Use consistency to facilitate recognition. Reduce the information presented to help with memory impairment problems.

- Clear indication of the current screen to avoid getting lost in the application.

- Use meaningful icons and labels, so that the users do not need to struggle with vision problems.

The main characteristic of iTVCare is to receive reminders trough the television set. Elderly and their caregivers have a user profile to set reminders (see Figure 2), and while they are watching television a reminder message would appear in the screen (see Figure 3), this reminder would stay on the screen until the user take action with it: "close the message" or "see more details". When the user chooses to "see more details", by tapping the notification message, a new windows appeared with the detailed information of the medication to take:

- Name of medication.

- Dose.

- Frequency of drug intake.

- Treatment duration.

- First intake.

The agenda manager uses the same interaction as the medication intakes. The information included in the detailed screen for the notification are:

- Name of appointment.

- Address.

- Date.

- Time.

- Additional information.

\subsection{Heuristic evaluation}

At this point, a heuristic evaluation study was performed through expert review to make sure that the requirements identified after the contextual study were included in a user-friendly manner to older adults.

The heuristic principles evaluated in the instrument were: 1) Visibility of system status, 2) Match between system and the real world, 3) User control and freedom, 4) Consistency and standards, 5) Error prevention, 6) Recognition rather than recall, 7) Flexibility and efficiency of use, 8) Aesthetic and minimalist design, 9) Help users recognize, diagnose, and recover from errors, 10) Help and documentation. The values for the ratings were between 0 (Not a problem) and 5 (Catastrophe).

The selected evaluators (5 and a pilot test) work in the Human-Computer Interaction area, with experience in the evaluation of interfaces and usability. The profile of the evaluators is shown in Table 1.

The experts were assigned with a list of task to perform with the prototype: 1) Add a new user. 2) Remove a user. 3) Add a 
medicine intake reminder. 4) Remove a medicine intake reminder. 5) Add a reminder for medical appointment. 6) Remove a reminder for medical appointment.

The experts identified 42 problems in the iTVCare prototype, of which $16(38.1 \%)$ were unique problems, that means, only one expert marked it as a problem. No problem was identified at the same time by all the evaluators. Each expert identified an average of 7 problems. The heuristics that were evaluated with the most problems are "Flexibility and efficiency of use" and "Help and documentation" which were identified by $87 \%$ and $67 \%$ of the experts, respectively.

Heuristics were an efficient method for finding problems with the system and allowed significant improvements in the interaction with the software [8].

\subsection{Technology acceptance evaluation}

After addressing and resolving the findings of the heuristic evaluation, an improved iTVCare platform was built (Android TV app). An assessment of technology adoption and user experience of the system was performed through a pilot test with a group of 50 seniors living in nursing homes in Mexico. The sample was selected by convenience sampling, per the availability of the subjects; the seniors belonged to two nursing homes at the city of Colima.

The purpose of the evaluation was to assess the technology acceptance of the iTVCare platform, to obtain evidence and determine if a television set is suitable and accepted by older adults to provide support in an age-friendly environment.

The intervention was started with an initial questionnaire for user characterization. The next step was to describe the system to the participants and a detailed demonstration of iTVCare in order to explain its functionality. Then, a set of task were assigned to be performed with the application, and finally, participants were asked to fill out the Technology Acceptance Model (TAM) questionnaire; this is a very common instrument to measure technology acceptance.

The findings confirmed that older adults showed a strong intention of use towards a television-based platform to provide wellbeing. Users had a good attitude for using it, generating a strong intention to use this platform to support their daily life activities.

\section{Potential Conclusions}

During last year, we focused on nursing homes environment to understanding the needs of older adult's groups (not only individuals) to inform an inclusive design of a set of guidelines to improve the interaction design of television applications to be used by a collective of elders.

A major contribution of this study was to validate the viability of the use of a TV set and applications (specially designed for TV) to support an ageing-friendly environment.

\section{Closing remarks}

Attendance to the doctoral consortium of MexIHC 2018 would be a great fit for this work, since the guidance of renowned experts would help in advance the project, further the learning and improve on this work. Also, this participation could help us on expanding our network connections to collaborate in future research projects.

\section{References}

[1] Chadia Abras, Diane Maloney-Krichmar, and Jenny Preece. User-Centered Design. In W. Bainbridge, ed., Encyclopedia of Human-Computer Interaction. Sage Publications, Thousand Oaks.

[2] Malek Alaoui and Myriam Lewkowicz. 2012. Struggling Against Social Isolation of the Elderly-The Design of SmartTV Applications. In J. Dugdale, C. Masclet, M.A. Grasso, J.-F. Boujut, and P. Hassanaly, eds., From Research to Practice in the Design of Cooperative Systems: Results and Open Challenges. Springer London, London, 261-275.

[3] Maris Kuningas, Reedik Mägi, Rudi G J Westendorp, P Eline Slagboom, Maido Remm, and Diana van Heemst. 2007. Haplotypes in the human Foxo1a and Foxo3a genes; impact on disease and mortality at old age. European Journal of Human Genetics 15, 3:294-301.

[4] Nursing Informatics. 2011. IT in Future Health and Social Care - Nurse Gudrun's Full-Scale Lab. Nursing Informatics Learning Center. Retrieved from https://goo.gl/Nmn4Kb.

[5] Carlos Rivas-Costa, Luis Anido-Rifon, and Manuel Jose Fernandez Iglesias. 2016. An Open Architecture to Support Social and Health Services in a Smart TV Environment. IEEE Journal of Biomedical and Health Informatics: 1-1.

[6] Marcela D. Rodríguez, Victor M. Gonzalez, Jesus Favela, and Pedro C. Santana. 2009. Home-based communication system for older adults and their remote family. Computers in Human Behavior 25, 3:609-618.

[7] Pedro C. Santana, Marcela D. Rodríguez, Víctor M. González, Luís A. Castro, and Ángel G. Andrade. 2005. Supporting emotional ties among Mexican elders and their families living abroad. ACM Press, 2099.

[8] Pedro Cesar Santana and Luis E Anido. 2016. Heuristic Evaluation of an Interactive Television System to Facilitate Elders Home Care. IEEE Latin America Transactions 14, 7 : 3455-3460.

[9] Pedro Santana-Mancilla and Luis Anido-Rifón. 2017. The Technology Acceptance of a TV Platform for the Elderly Living Alone or in Public Nursing Homes. International Journal of Environmental Research and Public Health 14, 6.

[10] Hélène Sauzéon, Bernard N'Kaoua, Prashant Arvind Pala, Mathieu Taillade, and Pascal Guitton. 2016. Age and active navigation effects on episodic memory: A virtual reality study. British Journal of Psychology 107, 1: 72-94.

[11] Hélène Sauzéon, Bernard N'Kaoua, Prashant Arvind Pala, Mathieu Taillade, Sophie Auriacombe, and Pascal Guitton. 2016. Everyday-like memory for objects in ageing and Alzheimer's disease assessed in a visually complex environment: The role of executive functioning and episodic memory. Journal of Neuropsychology 10, 1: 33-5 